\title{
Drell-Yan Production at Threshold to Third Order in QCD
}

\author{
Taushif Ahmed, ${ }^{1, *}$ Maguni Mahakhud, ${ }^{1, \dagger}$ Narayan Rana, ${ }^{1, \ddagger}$ and V. Ravindran ${ }^{2, \S}$ \\ ${ }^{1}$ RECAPP, Harish-Chandra Research Institute, Allahabad, India \\ ${ }^{2}$ The Institute of Mathematical Sciences, Chennai, India
}

(Received 6 May 2014; published 11 September 2014)

\begin{abstract}
The recent computation on the full threshold contributions to Higgs boson production at next to next to next to leading order $\left(\mathrm{N}^{3} \mathrm{LO}\right)$ in QCD contains valuable information on the soft gluons resulting from virtual and real emission partonic subprocesses. We use those from the real emissions to obtain the corresponding soft gluon contributions to Drell-Yan production and determine the missing $\delta(1-z)$ part of the $\mathrm{N}^{3} \mathrm{LO}$. The numerical impact of threshold effects demonstrates the importance of our results in the precision study with the Drell-Yan process at the LHC.
\end{abstract}

DOI: 10.1103/PhysRevLett.113.112002

PACS numbers: $12.38 . \mathrm{Bx}$

Discovery of the Higgs boson and the exclusion limits on its mass strongly depend on the precise knowledge of its production mechanism in the standard model (SM) and its extensions. The fixed as well as resummed next to next to leading order (NNLO) [1] and leading log (NNLL) [2] quantum chromodynamics (QCD) corrections supplemented with two-loop electroweak effects [3] played an important role in the discovery of the Higgs boson by ATLAS and CMS collaborations [4] at the Large Hadron Collider. Also for Drell-Yan (DY) production, NNLO [5] and NNLL [6] QCD results are known. There have been several attempts to go beyond the NNLO level in QCD. The gluon and quark form factors [7-9], the mass factorization kernels [10], and the renormalization constant [11] for the effective operator describing the coupling of the Higgs boson with the SM fields in the infinite top quark mass limit up to the three-loop level in dimensional regularization, with space-time dimension $d=4+\epsilon$, enabled one to obtain the next to next to next to leading order $\left(\mathrm{N}^{3} \mathrm{LO}\right)$ threshold effects $[12,13]$, often called soft plus virtual (SV) contributions, to the inclusive Higgs boson and DY productions at the LHC, excluding the term proportional to $\delta(1-z)$ where the scaling parameter $z=m_{H}^{2} / \hat{s}$ for the Higgs boson and $z=m_{l^{+} l^{-}}^{2} / \hat{s}$ for DY. Here $m_{H}, m_{l^{+} l^{-}}$, and $\hat{s}$ are the mass of the Higgs boson, invariant mass of the dileptons, and center of mass energy of the partonic reaction responsible for production mechanism, respectively. Note that the finite mass factorized threshold contribution to the inclusive production cross section is expanded in terms of $\delta(1-z)$ and $\mathcal{D}_{i}(z)$, where

$$
\mathcal{D}_{i}(z)=\left(\frac{\ln ^{i}(1-z)}{(1-z)}\right)_{+}
$$

The $\delta(1-z)$ part of $\mathrm{N}^{3} \mathrm{LO}$ threshold contribution was not known until recently because of the lack of information on the complete soft contributions coming from real radiation processes, while the exact two- [14] and three-loop [9] quark and gluon form factors and NNLO soft contributions [15] to all orders in $\epsilon$ are already known. The full threshold $\mathrm{N}^{3} \mathrm{LO}$ result for Higgs boson production has now become reality due the recent computation by Anastasiou et al. [16] who have computed all these soft effects resulting from gluon radiations, which constitute the missing part. In this Letter, we investigate the impact of these soft gluon contributions on the $\delta(1-z)$ part of the $\mathrm{N}^{3} \mathrm{LO}$ to DY production. This completes the full $\mathrm{N}^{3} \mathrm{LO}$ threshold contribution to DY production.

The production cross section of a heavy particle, namely, a Higgs boson or a pair of leptons at the hadron colliders can be computed using

$$
\begin{aligned}
\sigma^{I}\left(s, q^{2}\right)= & \sum_{a b} \int d x_{1} d x_{2} f_{a}\left(x_{1}, \mu_{F}^{2}\right) f_{b}\left(x_{2}, \mu_{F}^{2}\right) \\
& \times \hat{\sigma}_{a b}^{I}\left(\hat{s}, q^{2}, \mu_{F}^{2}\right),
\end{aligned}
$$

where $\hat{s}=x_{1} x_{2} s, s$ is the hadronic center of mass energy, and $\hat{\sigma}_{a b}^{I}$ is the partonic cross section with initial state partons $a$ and $b . I=g$ for Higgs boson production with $q^{2}=m_{H}^{2}$ and $I=q$ for DY production with invariant mass of the dileptons being $q^{2} . \mu_{F}$ is the factorization scale. The threshold contribution at the partonic level, denoted by $\Delta_{I}^{\mathrm{SV}}\left(z, q^{2}, \mu_{R}^{2}, \mu_{F}^{2}\right)$, normalized by the Born cross section $\hat{\sigma}_{a b}^{I,(0)}$ times the Wilson coefficient $C_{W}^{I}\left(\mu_{R}^{2}\right)$, is given by

$$
\Delta_{I}^{\mathrm{SV}}\left(z, q^{2}, \mu_{R}^{2}, \mu_{F}^{2}\right)=\left.\mathcal{C} \exp \left(\Psi^{I}\left(z, q^{2}, \mu_{R}^{2}, \mu_{F}^{2}, \epsilon\right)\right)\right|_{\epsilon=0},
$$

where $\mu_{R}$ is the renormalization scale, the dimensionless variable $z=q^{2} / \hat{s}$, and $\Psi^{I}\left(z, q^{2}, \mu_{R}^{2}, \mu_{F}^{2}, \epsilon\right)$ is a finite distribution. The symbol $\mathcal{C}$ implies convolution with the following expansion:

$$
\mathcal{C} e^{f(z)}=\delta(1-z)+\frac{1}{1 !} f(z)+\frac{1}{2 !} f(z) \otimes f(z)+\cdots
$$


Here $\otimes$ means Mellin convolution and $f(z)$ is a distribution of the kind $\delta(1-z)$ and $\mathcal{D}_{i}$. In $d=4+\epsilon$ dimensions,

$$
\begin{aligned}
\Psi^{I}\left(z, q^{2}, \mu_{R}^{2}, \mu_{F}^{2}, \epsilon\right)= & \left\{\ln \left[Z^{I}\left(\hat{a}_{s}, \mu_{R}^{2}, \mu^{2}, \epsilon\right)\right]^{2}\right. \\
& \left.+\ln \left|\hat{F}^{I}\left(\hat{a}_{s}, Q^{2}, \mu^{2}, \epsilon\right)\right|^{2}\right\} \delta(1-z) \\
& +2 \Phi^{I}\left(\hat{a}_{s}, q^{2}, \mu^{2}, z, \epsilon\right) \\
& -2 \mathcal{C} \ln \Gamma_{I I}\left(\hat{a}_{s}, \mu^{2}, \mu_{F}^{2}, z, \epsilon\right),
\end{aligned}
$$

where $\mu$ is the scale introduced to define the dimensionless coupling constant $\hat{a}_{s}=\hat{g}_{s}^{2} / 16 \pi^{2}$ in dimensional regularization, $Q^{2}=-q^{2}, Z^{I}\left(\hat{a}_{s}, \mu_{R}^{2}, \mu^{2}, \epsilon\right)$ is the overall operator renormalization constant, which satisfies

$$
\mu_{R}^{2} \frac{d}{d \mu_{R}^{2}} \ln Z^{I}\left(\hat{a}_{s}, \mu_{R}^{2}, \mu^{2}, \epsilon\right)=\sum_{i=1}^{\infty} a_{s}^{i}\left(\mu_{R}^{2}\right) \gamma_{i-1}^{I},
$$

where $a_{s}\left(\mu_{R}^{2}\right)$ is the renormalized coupling constant that is related to $\hat{a}_{s}$ through strong coupling constant renormalization $Z\left(a_{s}\left(\mu_{R}^{2}\right)\right)$, that is $\hat{a}_{s}=\left(\mu / \mu_{R}\right)^{\epsilon} Z\left(\mu_{R}^{2}\right) S_{\epsilon}^{-1} a_{s}\left(\mu_{R}^{2}\right)$, $S_{\epsilon}=\exp \left[\left(\gamma_{E}-\ln 4 \pi\right) \epsilon / 2\right]$. Because of the gauge and renormalization group invariance, the bare form factors $\hat{F}^{I}\left(\hat{a}_{s}, Q^{2}, \mu^{2}, \epsilon\right)$ satisfy the following differential equation [17]:

$Q^{2} \frac{d}{d Q^{2}} \ln \hat{F}^{I}=\frac{1}{2}\left[K^{I}\left(\hat{a}_{s}, \frac{\mu_{R}^{2}}{\mu^{2}}, \epsilon\right)+G^{I}\left(\hat{a}_{s}, \frac{Q^{2}}{\mu_{R}^{2}}, \frac{\mu_{R}^{2}}{\mu^{2}}, \epsilon\right)\right]$,

where $K^{I}$ contains all the poles in $\epsilon$ and $G^{I}$ contains the terms finite in $\epsilon$. Renormalization group invariance of $\hat{F}^{I}\left(\hat{a}_{s}, Q^{2}, \mu^{2}, \epsilon\right)$ gives

$$
\mu_{R}^{2} \frac{d}{d \mu_{R}^{2}} K^{I}=-\mu_{R}^{2} \frac{d}{d \mu_{R}^{2}} G^{I}=-\sum_{i=1}^{\infty} a_{s}^{i}\left(\mu_{R}^{2}\right) A_{i}^{I} .
$$

$A_{i}^{I}$ 's are the cusp anomalous dimensions. Expanding the $\mu_{R}^{2}$ independent part of the solution of the renormalization group (RG) equation for $G^{I}, G^{I}\left(a_{s}\left(Q^{2}\right), 1, \epsilon\right)=$ $\sum_{i=1}^{\infty} a_{s}^{i}\left(Q^{2}\right) G_{i}^{I}(\epsilon)$, one finds that $G_{i}^{I}$ can be decomposed in terms of collinear $B_{i}^{I}$ and soft $f_{i}^{I}$ anomalous dimensions as follows [18]:

$$
G_{i}^{I}(\epsilon)=2\left(B_{i}^{I}-\gamma_{i}^{I}\right)+f_{i}^{I}+C_{i}^{I}+\sum_{k=1}^{\infty} \epsilon^{k} g_{i}^{I, k},
$$

where $C_{1}^{I}=0, C_{2}^{I}=-2 \beta_{0} g_{1}^{I, 1}, C_{3}^{I}=-2 \beta_{1} g_{1}^{I, 1}-2 \beta_{0}\left(g_{2}^{I, 1}+\right.$ $\left.2 \beta_{0} g_{1}^{I, 2}\right), C_{4}^{I}=-2 \beta_{2} g_{1}^{I, 1}-2 \beta_{1}\left(g_{2}^{I, 1}+4 \beta_{0} g_{1}^{I, 2}\right)-2 \beta_{0}\left(g_{3}^{I, 1}+\right.$ $\left.2 \beta_{0} g_{2}^{I, 2}+4 \beta_{0}^{2} g_{1}^{I, 3}\right)$, and $\beta_{i}$ are the coefficients of the $\beta$ function of strong coupling constant $a_{s}\left(\mu_{R}^{2}\right), \mu_{R}^{2} d a_{s}\left(\mu_{R}^{2}\right) /$ $d \mu_{R}^{2}=\epsilon a_{s}\left(\mu_{R}^{2}\right) / 2-\sum_{i=0}^{\infty} \beta_{i} a_{s}^{i+2}\left(\mu_{R}^{2}\right)$. The coefficients $g_{i}^{I, k}$ can be obtained from the form factors. $G_{1}^{I}(\epsilon)$ and $G_{2}^{I}(\epsilon)$ are known to all orders in $\epsilon$ and $G_{3}^{I}(\epsilon)$ is known to $O\left(\epsilon^{3}\right)$ [9].

The mass factorization kernel $\Gamma\left(z, \mu_{F}^{2}, \epsilon\right)$ removes the collinear singularities which arise due to massless partons and it satisfies the following RG equation:

$$
\mu_{F}^{2} \frac{d}{d \mu_{F}^{2}} \Gamma\left(z, \mu_{F}^{2}, \epsilon\right)=\frac{1}{2} P\left(z, \mu_{F}^{2}\right) \otimes \Gamma\left(z, \mu_{F}^{2}, \epsilon\right) .
$$

$P\left(z, \mu_{F}^{2}\right)$ are Altarelli-Parisi splitting functions. In perturbative QCD, $P\left(z, \mu_{F}^{2}\right)=\sum_{i=1}^{\infty} a_{s}^{i}\left(\mu_{F}^{2}\right) P^{(i-1)}(z)$. We find that only diagonal elements of the kernel, $\Gamma_{I I}\left(\hat{a}_{s}, \mu_{F}^{2}\right.$, $\left.\mu^{2}, z, \epsilon\right)$ contribute to threshold corrections because they contain $\delta(1-z)$ and $\mathcal{D}_{0}$ at every order perturbation theory while the nondiagonal ones are regular functions in $z$, that is, $P_{I I}^{(i)}(z)=2\left[B_{i+1}^{I} \delta(1-z)+A_{i+1}^{I} \mathcal{D}_{0}\right]+P_{\text {reg, }, I}^{(i)}(z)$.

The finiteness of $\Delta_{I}^{\mathrm{SV}}$ demands that the soft distribution function $\Phi^{I}\left(\hat{a}_{s}, q^{2}, \mu^{2}, z, \epsilon\right)$ also satisfies Sudakov-type differential equations [13], namely,

$$
\begin{aligned}
q^{2} \frac{d}{d q^{2}} \Phi^{I}= & \frac{1}{2}\left[\bar{K}^{I}\left(\hat{a}_{s}, \frac{\mu_{R}^{2}}{\mu^{2}}, z, \epsilon\right)\right. \\
& \left.+\bar{G}^{I}\left(\hat{a}_{s}, \frac{q^{2}}{\mu_{R}^{2}}, \frac{\mu_{R}^{2}}{\mu^{2}}, z, \epsilon\right)\right] .
\end{aligned}
$$

$\bar{K}^{I}$ and $\bar{G}^{I}$ take the forms similar to those of $K^{I}$ and $G^{I}$ of the form factors in such a way that $\Psi^{I}$ is finite as $\epsilon \rightarrow 0$. The solution to the above equation is found to be

$$
\Phi^{I}=\sum_{i=1}^{\infty} \hat{a}_{s}^{i}\left(\frac{q^{2}(1-z)^{2}}{\mu^{2}}\right)^{i \frac{\epsilon}{2}} S_{\epsilon}^{i}\left(\frac{i \epsilon}{1-z}\right) \hat{\phi}^{I,(i)}(\epsilon),
$$

where $\hat{\phi}^{I,(i)}(\epsilon)=\left[\bar{K}^{I,(i)}(\epsilon)+\bar{G}^{I,(i)}(\epsilon)\right] / i \epsilon$ and $\mu_{R}^{2} d \bar{K}^{I} / d \mu_{R}^{2}=$ $-\delta(1-z) \mu_{R}^{2} d K^{I} / d \mu_{R}^{2}$. This implies that $\bar{K}^{I,(i)}(\epsilon)$ can be written in terms of $A_{i}^{I}$ and $\beta_{i}$. We define $\overline{\mathcal{G}}_{i}^{I}(\epsilon)$ through

$$
\sum_{i=1}^{\infty} \hat{a}_{s}^{i}\left(\frac{q_{z}^{2}}{\mu^{2}}\right)^{i \frac{e}{2}} S_{\epsilon}^{i} \bar{G}^{I,(i)}(\epsilon)=\sum_{i=1}^{\infty} a_{s}^{i}\left(q_{z}^{2}\right) \overline{\mathcal{G}}_{i}^{I}(\epsilon)
$$

where $q_{z}^{2}=q^{2}(1-z)^{2}$. Using the fact that $\Delta_{I}^{\mathrm{SV}}$ is finite as $\epsilon \rightarrow 0$, we can express $\overline{\mathcal{G}}_{i}^{I}(\epsilon)$ in the form similar to that of $G_{i}^{I}(\epsilon)$ by setting $\gamma_{i}^{I}=0, B_{i}^{I}=0$ and replacing $f_{i}^{I} \rightarrow-f_{i}^{I}$ and $g_{i}^{I, j} \rightarrow \overline{\mathcal{G}}_{i}^{I, j}$. The unknown constants $\overline{\mathcal{G}}_{i}^{I, j}$ can be extracted from the soft part of the partonic reactions. Since $\Phi^{I}$ results from the soft radiations, the constants $\overline{\mathcal{G}}_{i}^{I}(\epsilon)$ are found to be maximally non-Abelian [13] satisfying

$$
\overline{\mathcal{G}}_{i}^{q}(\epsilon)=\frac{C_{F}}{C_{A}} \overline{\mathcal{G}}_{i}^{g}(\epsilon),
$$

with $C_{A}=N, C_{F}=\left(N^{2}-1\right) / 2 N, N$ is the number of colors. Equation (9) implies that the entire soft distribution function for the DY production can be obtained from that 
of Higgs boson production. Substituting $Z^{I}$, the solutions for both $\hat{F}^{I}$ and $\Phi^{I}$, and $\Gamma_{I I}$ in Eq. (3), we obtain $\Delta_{I}^{\mathrm{SV}}$ in powers of $a_{s}\left(\mu_{R}^{2}\right)$ as

$$
\begin{aligned}
\Delta_{I}^{\mathrm{SV}}(z) & =\sum_{i=0}^{\infty} a_{s}^{i}\left(\mu_{R}^{2}\right) \Delta_{I, i}^{\mathrm{SV}}\left(z, \mu_{R}^{2}\right), \quad \text { where } \\
\Delta_{I, i}^{\mathrm{SV}} & =\left.\Delta_{I, i}^{\mathrm{SV}}\left(\mu_{R}^{2}\right)\right|_{\delta} \delta(1-z)+\left.\sum_{j=0}^{2 i-1} \Delta_{I, i}^{\mathrm{SV}}\left(\mu_{R}^{2}\right)\right|_{\mathcal{D}_{j}} \mathcal{D}_{j} .
\end{aligned}
$$

We have set $\mu_{R}^{2}=\mu_{F}^{2}=q^{2}$ and their dependence can be retrieved using the appropriate renormalization group equation. $\Delta_{I, i}^{\mathrm{SV}}\left(Q^{2}\right)$ are finite and they depend on the anomalous dimensions $A_{i}^{I}, B_{i}^{I}, f_{i}^{I}$ and $\gamma_{i}^{I}$, the $\beta$ function coefficients $\beta_{i}$ and $\epsilon$ expansion coefficients of $G^{I}(\epsilon), g_{j}^{I, i}$, s and of the corresponding $\overline{\mathcal{G}}^{I}(\epsilon), \overline{\mathcal{G}}_{j}^{I, i}$ s. Up to the two-loop level, all these terms are known to sufficient accuracy to obtain $\Delta_{I, 1}^{\mathrm{SV}}$ and $\Delta_{I, 2}^{\mathrm{SV}}$ exactly. At next to next to next to leading order level, only $\left.\Delta_{I, 3}^{\mathrm{SV}}\right|_{\mathcal{D}_{i}}$ 's were known $[12,13]$ as the term $\overline{\mathcal{G}}_{3}^{g, 1}$ needed for $\left.\Delta_{I, 3}^{\mathrm{SV}}\right|_{\delta}$ has not been available. Recently in Ref. [16], Anastasiou et al. have obtained $\left.\Delta_{g, 3}^{\mathrm{SV}}\right|_{\delta}$, using this we extract $\overline{\mathcal{G}}_{3}^{g, 1}$. This along with Eq. (9) can be used to determine the corresponding $\overline{\mathcal{G}}_{3}^{q, 1}$ and hence $\left.\Delta_{q, 3}^{\mathrm{SV}}\right|_{\delta}$. This completes the evaluation of full DY soft plus virtual contributions at $\mathrm{N}^{3} \mathrm{LO}$. The result for $\overline{\mathcal{G}}_{3}^{I, 1}$ is given by

$$
\begin{aligned}
\overline{\mathcal{G}}_{3}^{I, 1}= & C_{I}\left\{C_{A}{ }^{2}\left(\frac{152}{63} \zeta_{2}{ }^{3}+\frac{1964}{9} \zeta_{2}{ }^{2}+\frac{11000}{9} \zeta_{2} \zeta_{3}-\frac{765127}{486} \zeta_{2}+\frac{536}{3} \zeta_{3}^{2}-\frac{59648}{27} \zeta_{3}-\frac{1430}{3} \zeta_{5}+\frac{7135981}{8748}\right)\right. \\
& +C_{A} n_{f}\left(-\frac{532}{9} \zeta_{2}^{2}-\frac{1208}{9} \zeta_{2} \zeta_{3}+\frac{105059}{243} \zeta_{2}+\frac{45956}{81} \zeta_{3}+\frac{148}{3} \zeta_{5}-\frac{716509}{4374}\right) \\
& +C_{F} n_{f}\left(\frac{152}{15} \zeta_{2}^{2}-88 \zeta_{2} \zeta_{3}+\frac{605}{6} \zeta_{2}+\frac{2536}{27} \zeta_{3}+\frac{112}{3} \zeta_{5}-\frac{42727}{324}\right) \\
& \left.+n_{f}{ }^{2}\left(\frac{32}{9} \zeta_{2}^{2}-\frac{1996}{81} \zeta_{2}-\frac{2720}{81} \zeta_{3}+\frac{11584}{2187}\right)\right\},
\end{aligned}
$$

with $n_{f}$ being the number of light flavors and $C_{I} \equiv C_{A}, C_{F}$ for $I=g, q$, respectively. The $\left.\Delta_{q, 3}^{\mathrm{SV}}\right|_{\delta}$ is given by

$$
\begin{aligned}
\left.\Delta_{q, 3}^{\mathrm{SV}}\right|_{\delta}= & C_{A}^{2} C_{F}\left(\frac{13264}{315} \zeta_{2}^{3}+\frac{14611}{135} \zeta_{2}^{2}-\frac{884}{3} \zeta_{2} \zeta_{3}+843 \zeta_{2}-\frac{400}{3} \zeta_{3}^{2}+\frac{82385}{81} \zeta_{3}-204 \zeta_{5}-\frac{1505881}{972}\right) \\
& +C_{A} C_{F}^{2}\left(-\frac{20816}{315} \zeta_{2}^{3}-\frac{1664}{135} \zeta_{2}^{2}+\frac{28736}{9} \zeta_{2} \zeta_{3}-\frac{13186}{27} \zeta_{2}+\frac{3280}{3} \zeta_{3}^{2}-\frac{20156}{9} \zeta_{3}-\frac{39304}{9} \zeta_{5}+\frac{74321}{36}\right) \\
& +C_{A} C_{F} n_{f}\left(-\frac{5756}{135} \zeta_{2}^{2}+\frac{208}{3} \zeta_{2} \zeta_{3}-\frac{28132}{81} \zeta_{2}-\frac{6016}{81} \zeta_{3}-8 \zeta_{5}+\frac{110651}{243}\right) \\
& +C_{F}^{3}\left(-\frac{184736}{315} \zeta_{2}^{3}+\frac{412}{5} \zeta_{2}^{2}+80 \zeta_{2} \zeta_{3}-\frac{130}{3} \zeta_{2}+\frac{10336}{3} \zeta_{3}^{2}-460 \zeta_{3}+1328 \zeta_{5}-\frac{5599}{6}\right) \\
& +C_{F}^{2} n_{f}\left(\frac{272}{135} \zeta_{2}^{2}-\frac{5504}{9} \zeta_{2} \zeta_{3}+\frac{2632}{27} \zeta_{2}+\frac{3512}{9} \zeta_{3}+\frac{5536}{9} \zeta_{5}-\frac{421}{3}\right) \\
& +C_{F} n_{f, v}\left(\frac{N^{2}-4}{N}\right)\left(-\frac{4}{5} \zeta_{2}^{2}+20 \zeta_{2}+\frac{28}{3} \zeta_{3}-\frac{160}{3} \zeta_{5}+8\right)+C_{F} n_{f}^{2}\left(\frac{128}{27} \zeta_{2}^{2}+\frac{2416}{81} \zeta_{2}-\frac{1264}{81} \zeta_{3}-\frac{7081}{243}\right)
\end{aligned}
$$

where, $n_{f, v}$ is proportional to the charge weighted sum of the quark flavors [9].

We present the contribution from $\left.\Delta_{q, 3}^{\mathrm{SV}}\right|_{\delta}$ to pure $\mathrm{N}^{3} \mathrm{LO}_{\mathrm{SV}}$ as $\delta_{\mathrm{N}^{3} \mathrm{LO}}$ and the contributions from $\left.\Delta_{q, 3}^{\mathrm{SV}}\right|_{\mathcal{D}_{i}} \mathrm{~S}$ to pure $\mathrm{N}^{3} \mathrm{LO}_{\mathrm{SV}}$ as $\mathcal{D}_{\mathrm{N}^{3} \mathrm{LO}}$ in the Table I for different invariant masses $\left(m_{l^{+} l^{-}} \equiv Q\right)$ of the dileptons. We have used $\sqrt{s}=$ $14 \mathrm{TeV}$ for the LHC, number of light quark flavors $n_{f}=5$, Fermi constant $G_{F}=4541.68 \mathrm{pb}$, the $Z$ boson mass
$m_{Z}=91.1876 \mathrm{GeV}$, and top quark mass $m_{t}=173.4 \mathrm{GeV}$ throughout. The strong coupling constant $\alpha_{s}\left(\mu_{R}^{2}\right)$ is evolved using the four-loop renormalization group equations with $\alpha_{s}^{\mathrm{N}^{3} \mathrm{LO}}\left(m_{Z}\right)=0.117$ and for parton density sets we use Martin-Stirling-Thorne-Watt (MSTW) 2008NNLO [19].

We find that the $\delta$ contribution is almost equal and opposite in sign to the sum of the contributions from the $\mathcal{D}_{i}$ 's. Hence, adding the $\delta$ part reduces the pure $\mathrm{N}^{3} \mathrm{LO}_{\mathrm{SV}}$ 
TABLE I. Contributions of $\delta_{\mathrm{N}^{3} \mathrm{LO}}, \mathcal{D}_{\mathrm{N}^{3} \mathrm{LO}}$, NNLO (SV), exact NNLO, $\mathrm{N}^{3} \mathrm{LO}(\mathrm{SV})$ and $\mathrm{N}^{3} \mathrm{LO}$ SV

\begin{tabular}{|c|c|c|c|c|c|c|c|c|c|c|}
\hline$Q(\mathrm{GeV})$ & 30 & 50 & 70 & 90 & 100 & 200 & 400 & 600 & 800 & 1000 \\
\hline $10^{3} \delta_{\mathrm{N}^{3} \mathrm{LO}}(\mathrm{nb})$ & 11.386 & 2.561 & 1.724 & 140.114 & 5.410 & $4.567 \times 10^{-2}$ & $3.153 \times 10^{-3}$ & $6.473 \times 10^{-4}$ & $2.006 \times 10^{-4}$ & $7.755 \times 10^{-5}$ \\
\hline $10^{3} \mathcal{D}_{\mathrm{N}^{3} \mathrm{LO}}(\mathrm{nb})$ & -8.397 & -2.053 & acs & -124.493 & & & & $-7.455 \times 10^{-4}$ & $-2.456 \times 10^{-4}$ & $-9.959 \times 10^{-5}$ \\
\hline NNLO (SV) & 0.497 & 0.147 & 0.117 & 10.749 & 0.436 & $4.917 \times 10^{-3}$ & $4.364 \times 10^{-4}$ & $1.032 \times 10^{-4}$ & $3.538 \times 10^{-5}$ & $1.480 \times 10^{-5}$ \\
\hline NNLO & 0.543 & 0.158 & 0.124 & 11.296 & 0.458 & $5.233 \times 10^{-3}$ & $4.694 \times 10^{-4}$ & $1.116 \times 10^{-4}$ & $3.836 \times 10^{-5}$ & $1.607 \times 10^{-5}$ \\
\hline $\mathrm{N}^{3} \mathrm{LO}(\mathrm{SV})$ & 0.500 & 0.148 & 0.118 & 10.765 & 0.436 & $4.918 \times 10^{-3}$ & $4.362 \times 10^{-4}$ & $1.032 \times 10^{-4}$ & $3.534 \times 10^{-5}$ & $1.478 \times 10^{-5}$ \\
\hline $\mathrm{N}^{3} \mathrm{LO}_{\mathrm{SV}}$ & 0.546 & 0.158 & 0.124 & 11.311 & 0.459 & $5.234 \times 10^{-3}$ & $4.692 \times 10^{-4}$ & $1.116 \times 10^{-4}$ & $3.832 \times 10^{-5}$ & $1.605 \times 10^{-5}$ \\
\hline
\end{tabular}

term to 1 order in magnitude, establishing the dominance of the $\delta$ term. We have studied the effect of threshold corrections resulting from distributions such as $\delta(1-z)$ and $\mathcal{D}_{i}$ both at NLO as well as NNLO levels. In the following, we report our findings based on the numerical analysis presented in the table for two different ranges of $Q$, namely, $Q=200-1000 \mathrm{GeV}$ (above $m_{Z}$ ) and 30-100 GeV (below $m_{Z}$ ). At NLO, if we keep only the distributions and drop contributions from hard radiations coming from $q \bar{q}$ and $q(\bar{q}) g$ initiated processes, we find that the resulting NLO corrected cross section is about $95 \%$ of the exact result at NLO level. Similarly, if we keep the distributions and drop all the hard radiations both in NLO as well as in NNLO terms, we find that the resulting $\mathrm{NNLO}$ corrected result $[\mathrm{NNLO}(\mathrm{SV})]$ is about $95 \%$ of the exact one at NNLO level. Hence, it is expected that the sum $\left(\mathrm{N}^{3} \mathrm{LO}_{\mathrm{SV}}\right)$ of threshold contributions of $\mathrm{N}^{3} \mathrm{LO}$ terms and the exact NNLO corrected result would constitute the dominant contribution at $\mathrm{N}^{3} \mathrm{LO}$ level. Like NNLO terms, the threshold contributions in $\mathrm{N}^{3} \mathrm{LO}$ terms are also moderate and hence the perturbation theory behaves well. In Fig. 1, we have shown the dependence of our result on the renormalization scale at various orders in perturbation theory. We have plotted $R^{(i)}=\sigma^{i\left(\mu_{R}\right)} / \sigma^{i(Q)}$ where $i=\mathrm{NLO}, \mathrm{NNLO}, \mathrm{N}^{3} \mathrm{LO}_{\mathrm{SV}}$ versus $\mu_{R} / Q$ and the reduction in the scale dependence is evident as we increase the order of perturbation.

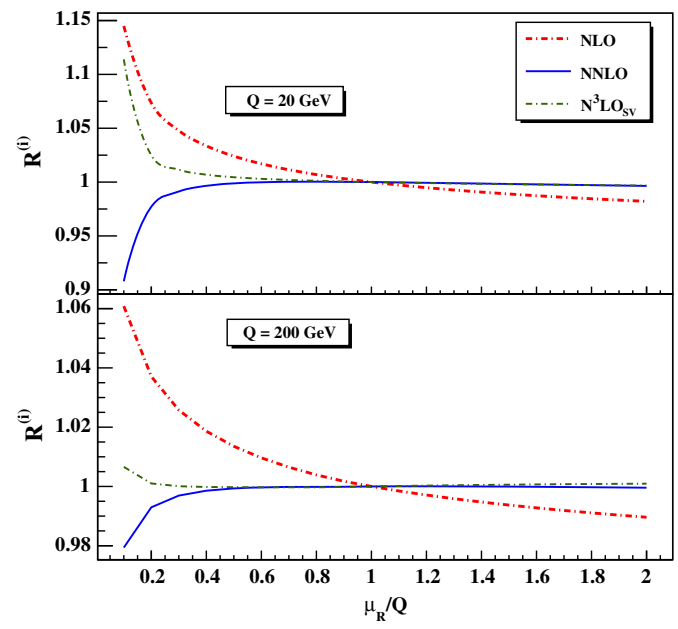

FIG. 1 (color online). Scale variation.
To summarize, we present a systematic way of computing the threshold corrections to inclusive Higgs boson and DY productions in perturbative QCD. We have used several properties of QCD amplitudes, namely, factorization of soft and collinear divergences, renormalization group invariance, and resummation of threshold contributions. For the first time we show that the recent $\mathrm{N}^{3} \mathrm{LO}$ soft plus virtual contribution to the Higgs boson production cross section can be used to obtain the corresponding $\delta(1-z)$ part of DY production at $\mathrm{N}^{3} \mathrm{LO}$. We also present numerical results to establish the importance of the $\delta$ term. We find that the impact of the $\delta$ contribution is quite large to the pure $\mathrm{N}^{3} \mathrm{LO}_{\mathrm{SV}}$ correction. We have also demonstrated the dominance of threshold corrections at every order in perturbation theory. We expect that the results presented in this Letter will not only be a benchmark for a full $\mathrm{N}^{3} \mathrm{LO}$ order contribution but also an important step in the precision study with the Drell-Yan process at the LHC.

T. A., M. M., and N. R. are thankful for the hospitality provided by the Institute of Mathematical Sciences (IMSc) where the work was carried out. We thank the staff of IMSc computer center for their help. We thank M. K. Mandal for his generous help. We also thank the referees for useful suggestions. The work of T. A., M. M., and N. R. has been partially supported by funding from RECAPP, Department of Atomic Energy, Government of India.

*taushif@hri.res.in

†maguni@hri.res.in

narayan@hri.res.in

§ravindra@imsc.res.in

[1] H. M. Georgi, S. L. Glashow, M. E. Machacek, and D. V. Nanopoulos, Phys. Rev. Lett. 40, 692 (1978); A. Djouadi, M. Spira, and P. M. Zerwas, Phys. Lett. B 264, 440 (1991); S. Dawson, Nucl. Phys. B359, 283 (1991); M. Spira, A. Djouadi, D. Graudenz, and P. M. Zerwas, Nucl. Phys. B453, 17 (1995); S. Catani, D. de Florian, and M. Grazzini, J. High Energy Phys. 05 (2001) 025; R. V. Harlander and W. B. Kilgore, Phys. Rev. D 64, 013015 (2001); R. V. Harlander and W. B. KilgorePhys. Rev. Lett. 88, 201801 (2002); C. Anastasiou and K. Melnikov, Nucl. Phys. B646, 220 (2002); V. Ravindran, J. Smith, and W. L. van Neerven, Nucl. Phys. B665, 325 (2003). 
[2] S. Catani, D. de Florian, M. Grazzini, and P. Nason, J. High Energy Phys. 07 (2003) 028.

[3] U. Aglietti, R. Bonciani, G. Degrassi, and A. Vicini, Phys. Lett. B 595, 432 (2004); S. Actis, G. Passarino, C. Sturm, and S. Uccirati, Phys. Lett. B 670,12 (2008).

[4] G. Aad et al. (ATLAS Collaboration), Phys. Lett. B 716, 1 (2012); S. Chatrchyan et al. (CMS Collaboration), Phys. Lett. B 716, 30 (2012).

[5] G. Altarelli, R. K. Ellis, and G. Martinelli, Nucl. Phys. B143, 521 (1978); B146, 544(E) (1978); T. Matsuura and W. L. van Neerven, Z. Phys. C 38, 623 (1988); T. Matsuura, S. C. van der Marck, and W. L. van Neerven, Nucl. Phys. B319, 570 (1989); R. Hamberg, W. L. van Neerven, and T. Matsuura, Nucl. Phys. B359, 343 (1991); B644, 403(E) (2002); R. V. Harlander and W. B. Kilgore, Phys. Rev. D 64, 013015 (2001).

[6] A. Vogt, Phys. Lett. B 497, 228 (2001).

[7] S. Moch, J. A. M. Vermaseren, and A. Vogt, J. High Energy Phys. 08 (2005) 049; Phys. Lett. B 625, 245 (2005).

[8] P. A. Baikov, K. G. Chetyrkin, A. V. Smirnov, V. A. Smirnov, and M. Steinhauser, Phys. Rev. Lett. 102, 212002 (2009).

[9] T. Gehrmann, E. W. N. Glover, T. Huber, N. Ikizlerli, and C. Studerus, J. High Energy Phys. 06 (2010) 094.
[10] S. Moch, J. A. M. Vermaseren, and A. Vogt, Nucl. Phys. B688, 101 (2004); B691, 129 (2004).

[11] K. G. Chetyrkin, B. A. Kniehl, and M. Steinhauser, Nucl. Phys. B510, 61 (1998).

[12] S. Moch and A. Vogt, Phys. Lett. B 631, 48 (2005); E. Laenen and L. Magnea, Phys. Lett. B 632, 270 (2006); A. Idilbi, X.-d. Ji, J.-P. Ma, and F. Yuan, Phys. Rev. D 73, 077501 (2006).

[13] V. Ravindran, Nucl. Phys. B746, 58 (2006); B752, 173 (2006).

[14] T. Gehrmann, T. Huber, and D. Maitre, Phys. Lett. B 622, 295 (2005).

[15] D. de Florian and J. Mazzitelli, J. High Energy Phys. 12 (2012) 088.

[16] C. Anastasiou, C. Duhr, F. Dulat, E. Furlan, T. Gehrmann, F. Herzog, and B. Mistlberger, arXiv:1403.4616.

[17] V. V. Sudakov, Zh. Eksp. Teor. Fiz. 30, 87 (1956) [Sov. Phys. JETP 3, 65 (1956)]; A. H. Mueller, Phys. Rev. D 20, 2037 (1979); J. C. Collins, Phys. Rev. D 22, 1478 (1980); A. Sen, Phys. Rev. D 24, 3281 (1981).

[18] V. Ravindran, J. Smith, and W. L. van Neerven, Nucl. Phys. B704, 332 (2005); T. Becher and M. Neubert, Phys. Rev. Lett. 102, 162001 (2009); E. Gardi and L. Magnea, J. High Energy Phys. 03 (2009) 079.

[19] A. D. Martin, W. J. Stirling, R. S. Thorne, and G. Watt, Eur. Phys. J. C 63, 189 (2009). 